\title{
Morphological study, production and cryopreservation of blastospores of entomopathogenic fungi
}

\author{
Lakkhana K. Wingfield* \\ Division of Biological Science, Faculty of Science, Prince of Songkla University, Songkhla 90110 Thailand \\ e-mail: Lakkhana.k@psu.ac.th
}

Received 11 Jul 2020

Accepted 7 Oct 2020

\begin{abstract}
Entomopathogenic fungi have been known as potential candidates for insecticides and metabolite producers. The aims of this study were to investigate characteristic and development patterns of blastospores and to determine optimal culture conditions for blastospore production of entomopathogenic fungi in the genera Akanthomyces, Cordyceps, Hirsutella, Metarhizium, and Torrubiella. Production of blastospores was induced using Grace's insect cell medium (GICM) supplemented with foetal bovine serum (FBS) except the genus Torrubiella. Out of 39 fungal isolates, 18 isolates could produce blastospores, most of them belonged to the genus Cordyceps. Observation on characteristics and formation of blastospores revealed that blastospores produced from all genera shared common characters and formation. However, some distinctive characters were observed in the genus Akanthomyces. Effects of culture media, types of inoculum and cultivating condition were examined for the production of highest numbers of blastospores (up to $10^{7}-10^{8}$ blastospores $/ \mathrm{ml}$ ), revealing different requirements in each fungal isolate for the production of blastospores. Cryopreservation of blastospores revealed that freeze-drying method could be used to preserved blastospores of C. brongniartii, Hisutella sp. 02 and Metarhizium sp. 02 except A. pistillariiformis. This study is the first comprehensive investigation on development patterns of blastospores and factors underpinning effective culture conditions for blastospores production of the selected entomopathogenic fungi.
\end{abstract}

KEYWORDS: entomopathogenic fungi, blastospores, cryopreservation

\section{INTRODUCTION}

Entomopathogenic fungi are important natural regulators of insect populations as they can generally infect a broad range of insects and cause mortality of the insect hosts. Since they are considered natural mortality agents and environmental safe, the fungi have been proposed as a group of potential candidates for microbial insecticides to control agricultural pests [1-4]. For example, Beauveria bassiana 36 was investigated for its virulence and pathogenicity on the infected larvae of Helicoverpa armigera, the most serious pest of several plant species, in the recent report [5]. Entomopathogenic fungi have also been signified as a valuable source of secondary bioactive metabolites. Several bioactive compounds have been isolated from Cordyceps spp., for instance, antimalarial cordypyridones A-D from C. nipponica $[6,7]$, bioxanthracenes from C. pseudomilitaris [7], antimalarial red naphthoquinones from C. unilateralis [8], and beauvericin-like compound from C. militaris [9].

The entomopathogenic fungi consist of various taxa and do not form a monophyletic group. Most of common and important entomopathogenic fungi are grouped in the order Hypocreales which belongs to the phylum Ascomycota. These include the anamorphic phases (Beauveria spp., Hirsutella spp., Metarhizium spp., Nomuraea spp., and Paecilomyces spp.) and the teleomorphic phase (Cordyceps spp.). Meanwhile, other entomopathogenic fungi (Entomophthora spp., Entomophaga spp., Pandora spp., and Zoophthora spp.) belong to the order Entomophthorales of the phylum Zygomycota. Mode of production of asexual and sexual propagules has been proposed as a key basis for identification of these fungi. Besides the vegetative propagules, other structures, such as rhizoids, resting spores, hyphal bodies (including protoplasts and blastospores), and sclerotia are also important for the classification of a fungal species or a genus [10]. Unlike other fungal groups, the entomopathogenic fungi can produce secondary spore-type which is commonly referred to blastospore $[11,12]$.

Formulation of microbial insecticides from entomopathogenic fungi often centers on massproduction of aerial conidia [1-3]. Interestingly, it has been evidenced that blastospores demonstrated 
stronger infection ability than conidia in terms of faster germination rate on the insect cuticle and higher level of bioactivity and insecticidal efficacy against various insect pests [13-15], which thus make blastospores the propagules of choice for the production of a biological insecticide. However, in vitro cultivation of blastospores is still very fastidious in some fungal genera because traditional culture conditions for fungal growth are unfavourable for the production of blastospores thus different parameters are required [16]. In addition, information on blastospore development and characteristics of individual entomopathogenic fungal genus is still sparse, which hinder the use of blastospores as microbial pesticides.

Since entomopathogenic fungi are a rich source of natural bioactive compounds and demonstrate certain advantages over the use of chemical insecticides, production of the microbial insecticides, particularly exploitation of the blastospore as an active ingredient, is challenging. In addition, understanding the production and development patterns of blastospores of the fungi is also crucial for developing blastospores as microbial insecticides. In response to those challenges, culture conditions for the production of blastospores, including blastospore development patterns, should be defined. The aim of the present study was to investigate characteristics and development patterns of blastospores of the selected entomopathogenic fungal genera. In addition, culture conditions to induce the production of blastospores were also determined. Besides difficulties in producing blastospores for morphological study and mass-production for industrial use, storage of fungal cultures is also very important that these cultures should be preserved in a physiologically and genetically stable state in order to maintain their valuable properties. Therefore, long-term preservation method via freeze-drying of the blastospores were also investigated.

\section{MATERIALS AND METHODS}

\section{Fungal isolates and maintenance}

Fungal isolates of the entomopathogenic fungi used in the present study were from the genera Akanthomyces, Cordyceps, Hirsutella, Metarhizium, and Torrubiella. All fungal isolates were maintained on Potato Dextrose Agar (PDA) (HiMedia ${ }^{\circledR}$ ) and incubated at $25^{\circ} \mathrm{C}$ for $10-15$ days.

\section{Induction and observation of blastospore development patterns and morphology}

Optimum concentrations of the foetal bovine serum (FBS; GIBCO ${ }^{\circledR}$, USA) used for the production of blastospores were determined. Ten entomopathogenic fungal isolates belonged to five fungal genera (Akanthomyces pistillariiformis, Akanthomyces sp. 01, Cordyceps brongniartii, Cordyceps sp. 01, Cordyceps sp. 02, Hirsutella sp. 01, Hirsutella sp. 02, Metarhizium sp. 01, Metarhizium sp. 02, Torrubiella sp. 01, and Torrubiella sp. 02) were selected for the study. A mycelial plug ( $0.5 \mathrm{~mm}$ in diameter) of each entomopathogenic fungal strain was transferred into $1 \mathrm{ml}$ of Grace's insect cell medium (GICM; GIBCO $®$, USA) containing $0.60 \mathrm{~g} / 1 \mathrm{~L}$-glutamine, $3.33 \mathrm{~g} / 1$ lactalbumin hydrolysate, $3.33 \mathrm{~g} / 1$ yeastolate, and $3 \%(\mathrm{w} / \mathrm{v})$ glucose and supplemented with different concentrations of FBS $(0,1,5$ and $10 \%(\mathrm{v} / \mathrm{v}))$ in a 24-well microtitre plate. The cultures were incubated at $28^{\circ} \mathrm{C}$ under static condition and the formation of blastospores was investigated daily for 14 days using inverted microscope. Formation, characteristics and morphological development of blastospores were observed daily using an inverted microscope.

\section{Large scale cultivation of blastospores}

To monitor rate of blastospore production, either conidia suspensions or mycelial plugs were used. Conidia suspensions $\left(10^{3}\right.$ conidia $\left./ \mathrm{ml}\right)$ were prepared using sterile distilled water containing $1 \%(\mathrm{v} / \mathrm{v})$ of tween 80 . Conidia suspensions $(200 \mu \mathrm{l})$ or 5 mycelial plugs, were transferred into $5 \mathrm{ml}$ of yeast extract-peptone-glucose (YPG) broth or GICM in a 6-well plate and incubated at $28^{\circ} \mathrm{C}$ under static condition and shaking condition at $100 \mathrm{rpm}$. The numbers of blastospores were counted using haemacytometer (Boeco, Germany). The experiment was conducted in triplicate. The conditions giving blastospore concentration of more than $10^{8}$ blastospores $/ \mathrm{ml}$ were selected for the production of blastospores to be used in the cryopreservation process.

\section{Cryopreservation of blastospores of entomopathogenic fungi}

Blastospores were filtered through double layers of sterile Miracloth and adjusted to desired concentration of $10^{7}-10^{8}$ blastospores $/ \mathrm{ml}$. Each blastospore suspension was centrifuged at $6600 \mathrm{rpm}$ for $5 \mathrm{~min}$ to remove the culture media and the pellet washed twice with sterile distilled water. The resulting blas- 
Table 1 Determination of FBS concentration for blastospore production (blastospores $/ \mathrm{ml}$ ).

\begin{tabular}{lcccc}
\hline \multirow{2}{*}{ Fungal isolate } & \multicolumn{4}{c}{ FBS concentration (\%) } \\
\cline { 2 - 5 } & 0 & 1 & 5 & 10 \\
\hline A. pistillariiformis & $10^{2}$ & $10^{5}$ & $10^{8}$ & $>10^{8}$ \\
Akanthomyces sp. 01 & - & - & - & - \\
C. brongniartii & $10^{5}$ & $10^{7}$ & $10^{8}$ & $>10^{8}$ \\
Cordyceps sp. 01 & $10^{4}$ & $10^{6}$ & $10^{7}$ & $10^{7}$ \\
Hirsutella sp. 01 & - & - & 10 & $10^{2}$ \\
Hirsutella sp. 02 & - & - & $10^{2}$ & $10^{4}$ \\
Metarhizium sp. 01 & - & - & $10^{2}$ & $10^{4}$ \\
Metarhizium sp. 02 & & 10 & $10^{4}$ & $10^{6}$ \\
Torrubiella sp. 01 & - & - & - & - \\
Torrubiella sp. 02 & - & - & - & - \\
\hline
\end{tabular}

tospore pellets were used within $2 \mathrm{~h}$ after washing. Protective agent ( $5 \%(\mathrm{w} / \mathrm{v})$ trehalose) was prepared in $0.1 \mathrm{M}$ potassium phosphate buffer $(\mathrm{pH} 7.0)$ and sterilized at $115^{\circ} \mathrm{C}$ for $15 \mathrm{~min}$. Blastospore pellet was resuspended in $0.1 \mathrm{ml}$ of the protective agent and filled in an ampoule. Primary vacuum drying was performed for the minimum of $3 \mathrm{~h}$, followed by secondary drying at $0.090 \mathrm{mbar}$ at $-60^{\circ} \mathrm{C}$ for $20 \mathrm{~h}$. Ampoules were sealed and stored at $5^{\circ} \mathrm{C}$ in the dark.

Blastospore viability was evaluated before drying, immediately after drying and after one-year storage. Freeze-dried blastospores were dispersed in $0.1 \mathrm{ml}$ rehydration fluid (peptone $5 \mathrm{~g}$, yeast extract $3 \mathrm{~g}, \mathrm{MgSO}_{4} 1 \mathrm{~g}$, and distilled water to $1 \mathrm{l}$, $\mathrm{pH}$ 7.0). Rehydrated blastospores were transferred into microtitre plate wells containing $1.5 \mathrm{ml}$ of GICM. After 8-15 h of incubation, one drop of $2 \mathrm{~N}$ $\mathrm{HCl}$ was added to halt the germination process. Germination of blastospores was assessed microscopically; and counting was done in duplicate from two ampoules. Revived cultures were also observed for morphological and genetic stability, such as changes in pigmentation or colony morphology.

\section{RESULTS}

\section{FBS concentration for the production of blastospores}

Ten entomopathogenic fungal isolates belonged to five fungal genera (Akanthomyces, Cordyceps, Hirsutella, Metarhizium, and Torrubiella) were selected for the study. Mycelial plugs of each isolate were grown in GICM supplemented with 0, 1, 5 and $10 \%(\mathrm{v} / \mathrm{v})$ FBS. The results revealed that blastospore production was observed from the genus Cordyceps and A. pistillariiformis without addition of FBS (Table 1). With addition of FBS, the production
Table 2 Screening of blastospore production from 39 entomopathogenic fungal isolates.

\begin{tabular}{lcc}
\hline Genera & $\begin{array}{l}\text { No. of isolates tested/ } \\
\text { No. of blastospore } \\
\text { producing isolates }\end{array}$ & $\begin{array}{l}\text { Frequency of } \\
\text { blastospore } \\
\text { production (\%) }\end{array}$ \\
\hline Akanthomyces & $9 / 3$ & 33 \\
Cordyceps & $16 / 10$ & 63 \\
Hirsutella & $5 / 3$ & 60 \\
Metarhizium & $5 / 2$ & 40 \\
Torrubiella & $4 / 0$ & 0 \\
\hline
\end{tabular}

of blastospores was significantly enhanced. FBS supplementation was necessary in the genera Hirsutella and Metarhizium as blastospore production was induced at high concentration of FBS (5 and $10 \%(\mathrm{v} / \mathrm{v}))$. Meanwhile, none of the Torrubiella and Akanthomyces sp. 01 isolates produced blastospores. This might infer specific preference of each fungal strain for blastospore production in vitro. Although $5 \%$ (v/v) FBS was sufficient for most isolates, $10 \%(\mathrm{v} / \mathrm{v})$ FBS was chosen for the entire study based on satisfactory blastospore yield.

\section{Screening for fungal isolates capable for blastospore production}

Production of blastospores was screened from 39 entomopathogenic fungi using GICM supplemented with $10 \%(\mathrm{v} / \mathrm{v})$ FBS. Out of 39 fungal isolates, 18 isolates were able to produce blastospores (Table 2). Most of them belonged to the genera Cordyceps and Hirsutella (10/16 and 3/5, respectively) while blastospore production was detected in few isolates of Akanthomyces and Metarhizium. In addition, none of Torrubiella isolates produced blastospores in GICM supplemented with $10 \%$ (v/v) FBS.

\section{General characteristics and formation patterns of blastospores}

Observation on characteristics and formation patterns of blastospores revealed that blastospores produced from all genera shared common characters and formation patterns (Table S1). However, some distinctive characters could be observed in some fungal isolates. In the Akanthomyces spp., blastospore formation was generally observed after 12 days of incubation. The spores were hyaline, small, round or oval shapes with 4-10 $\mu \mathrm{m}$ average in length. They were usually produced from hyphal tips and formed clusters. After released into the medium, blastospores immediately produced a new budding cell. In addition, it can be observed that 
Akanthomyces spp. demonstrated unique characteristics of blastospores that differed from other genera in terms of shape, size and budding position on the hypha. In the Cordyceps spp., blastospore formation was generally observed after 2-3 days of incubation. The spores were hyaline, ellipsoidal to cylindrical shapes which connected to the mycelium by a short neck. Average length of blastospore was 10-25 $\mu \mathrm{m}$. In addition, the spores usually produced from hyphal tips and sometimes from sides. In the Hirsutella spp., blastospore formation was generally observed after 2-5 days after inoculation depending on fungal strains. The spores were mainly hyaline, small, narrow with cylindrical to club-shapes. However, some blastospores were ellipsoidal, cylindrical to long rod shapes. The length of blastospores was between 15-20 $\mu \mathrm{m}$. Blastospores of Hirsutella were found to be generated very rapidly by budding both from the mother cells and from the hyphal sides. In the Metarhizium spp., blastospore formation was generally observed after 2-3 days of incubation. The spores were hyaline, ellipsoidal to cylindrical shapes and usually produced from hyphal sides with an attachment through a short neck. Average length of blastospore was 10-20 $\mu \mathrm{m}$.

\section{Detailed morphological development patterns of blastospores}

Representative isolates of each fungal genus (C. brongniartii, Hisutella sp. 02, Metarhizium sp. 02, and A. pistillariiformis) were chosen as model organisms, based on their ability to produce large numbers of blastospores and their industrial importance, to study the morphological development of blastospores.

C. brongniartii is one of the most important species among Cordyceps spp. This fungus can produce cuticle-degrading peptidases, which can potentially be used in agricultural and medical applications. Colony appearance of $C$. brongniartii grown on PDA at $28^{\circ} \mathrm{C}$ for one week were woolly to powdery with approximately $19.3 \pm 1.6 \mathrm{~mm}$ in diameter (Fig. 1a). Colony color was white to cream and yellowish-brown to dark brown on the reverse side resemble to its anamorph. After three weeks of incubation, the colony changed to brown and slender stalks of stromata were produced on the colony (Fig. 1b). Conidia were abundant, elliptical, with thick smooth walls and borne along the sides and ends of repeatedly branched hyphae to form large clusters. Size of conidia was $10.3 \pm 1.4 \mu \mathrm{m} \times 4.7 \pm 0.5 \mu \mathrm{m}$ (Fig. 1c). Blastospore formation of $C$. brongniartii was observed daily in GICM supplemented with $10 \%$ (v/v) FBS and the developmental process was initially examined from the stage of conidia development. The onset of conidia germination was marked by gradual swelling of conidia until it was approximately $30-100 \%$ larger than the original size after $8 \mathrm{~h}$ of incubation (Fig. 1d). After that the emergence of unipolar germ tubes were detected at $12 \mathrm{~h}$. Further elongation of the germ tubes and septation of hypha were apparent after $16 \mathrm{~h}$ of incubation (Fig. 1e). During this stage, bipolar or multipolar growth of young mycelia from the conidium occurred. After elongation of mycelia, a blastospore appeared laterally or sometimes terminally from mycelia after $24 \mathrm{~h}$ of incubation. Blastospores were connected to mycelium by a short neck. These spores propagated by production through a pore and bulged out near the septa. Release of free blastospores occurred after $36 \mathrm{~h}$ of incubation (Fig. 1f). At this stage, blastospores were hyaline, singlecelled with thin smooth-wall resemble protoplastlike cells. Detached blastospores were mostly elliptical shape. The average sizes of blastospores were $10.9-23.4 \mu \mathrm{m} \times 3.1-3.2 \mu \mathrm{m}$. Then, the spores' elongation proceeded and a cross-walled was made to separate part of the stretched blastospores after $48 \mathrm{~h}$ of incubation (Fig. 1g). These short mycelia derived from blastospores could reproduce new blastospores on the third day of cultivation (Fig. 1(h,i,j)). New generation of blastospores could be obtained from reproduction of newly grown mycelia.

Colony appearance of Hirsutella sp. 02 grown on PDA at $28^{\circ} \mathrm{C}$ for 7 days was flat with short aerial mycelia and moist colony surface with approximately $14.24 \pm 1.13 \mathrm{~mm}$ in diameter (Fig. 2a). Colony color was cream to golden brown and dark brown on the reverse side. Blastospores were hyaline, single-celled with smooth-wall and appeared laterally or sometimes terminally from mycelia after 2 days of incubation (Fig. 2b). The release of free blastospores was observed after 2.5 days of incubation. Detached blastospores were mostly elliptical to rod shape. The average size of detached blastospores was 11.1-22.6 $\mu \mathrm{m} \times 3.6-4.0 \mu \mathrm{m}$ (Fig. 2c). After release of blastospores, the spores were elongated and a cross wall was made to separate part of the stretched blastospores after 3.5 days of incubation (Fig. 2d). However, these short mycelia derived from blastospores could not reproduce new blastospores as observed in C. brongniartii. In this case, new blastospores were generated from budding of the mature blastospores only.

Metarhizium spp. is one of the most potential 


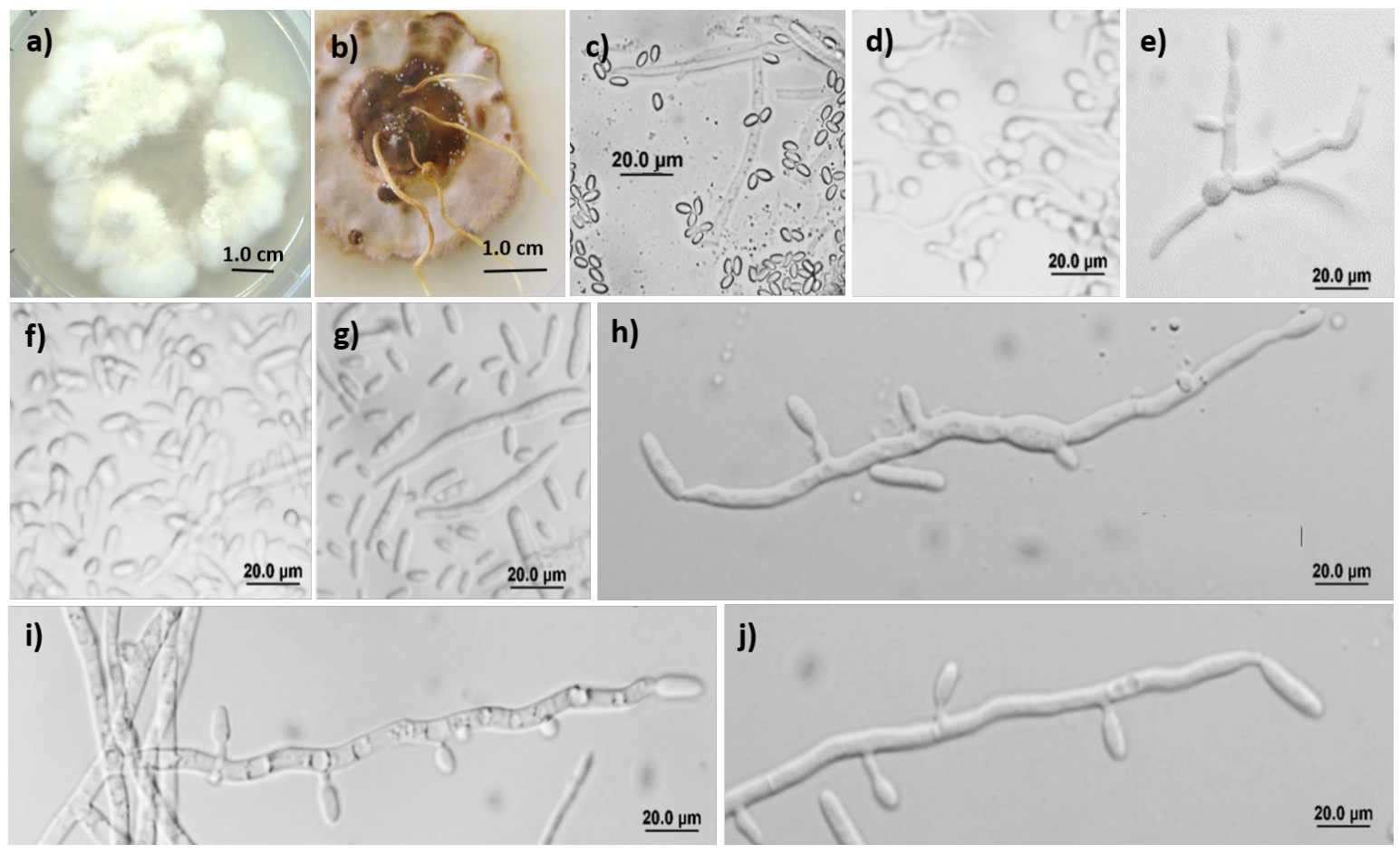

Fig. 1 Characteristics and morphological development patterns of blastospores of $C$. brongniartii. The liquid culture was grown in GICM supplemented with $10 \% \mathrm{FBS}$ and incubated at $28^{\circ} \mathrm{C}$ under static condition. (a) Colony of $C$. brongniartii on PDA incubated at $28^{\circ} \mathrm{C}$ for 7 days; (b) colony after incubation for 3 weeks; (c) conidia; (d) gradual swelling of conidia and emergence of a unipolar germ tube at 8-12 h; (e) blastospore formation from germinating conidia at 16$24 \mathrm{~h}$; (f) released blastospores at $36 \mathrm{~h}$; (g) germination and elongation of blastospores at $48 \mathrm{~h}$; (h,i,j) generation of blastospores from a mature mycelium at $54 \mathrm{~h}$.

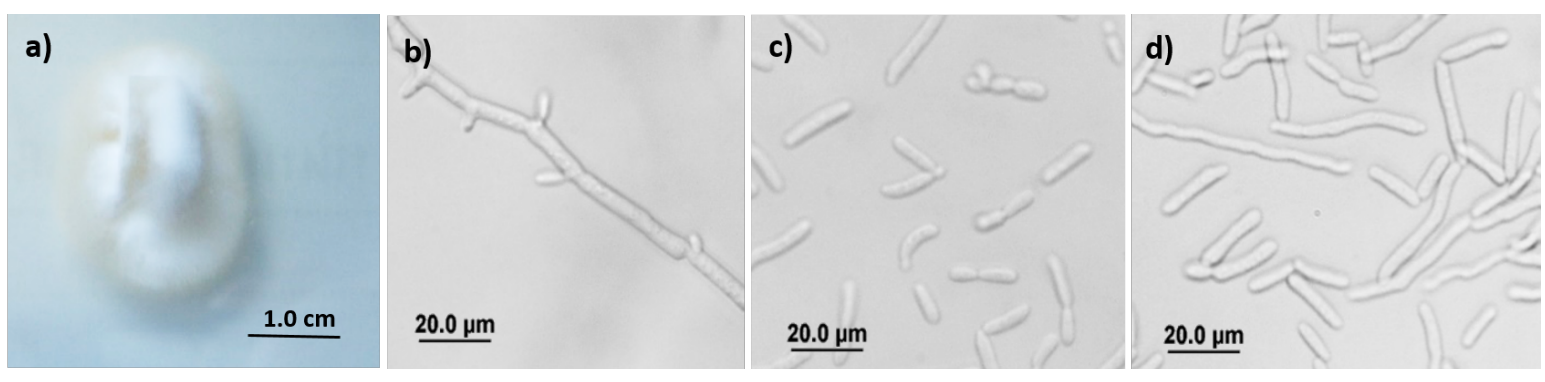

Fig. 2 Characteristics and morphological development patterns of blastospores of Hirsutella sp. 02. The liquid culture was grown in GICM supplemented with $10 \%$ FBS and incubated at $28^{\circ} \mathrm{C}$ under static condition. (a) Colony of Hirsutella sp. 02 on PDA incubated at $28^{\circ} \mathrm{C}$ for 7 days; (b) blastospore formation after 2 days of incubation; (c) released blastospores after 2.5 days of incubation; (d) germination and elongation of blastospores after 3.5 days of incubation.

entomopathogenic fungi which is used as biological insecticide to control a number of pests, such as grasshoppers, termites, thrips, etc. Colony appearance of Metarhizium sp. 02 grown on PDA at $28^{\circ} \mathrm{C}$ for one week was flat and velvety with approximately $22.61 \pm 2.15 \mathrm{~mm}$ in diameter (Fig. 3a). Colony color was white to creamy and yellow to orange on the reverse side. Conidia were single-celled, cylindrical or ovoid, forming chains (Fig. 3b). Blastospores were observed after 2 days of cultivation and appeared laterally or sometimes terminally from mycelia. They were hyaline, single-celled with smooth-wall (Fig. 3cd). Detached blastospores were mostly elliptical to rod shape with the average size of $15.0-21.3 \mu \mathrm{m} \times 3.6-4.7 \mu \mathrm{m}$. After released into the medium, enlargement of blastospores oc- 

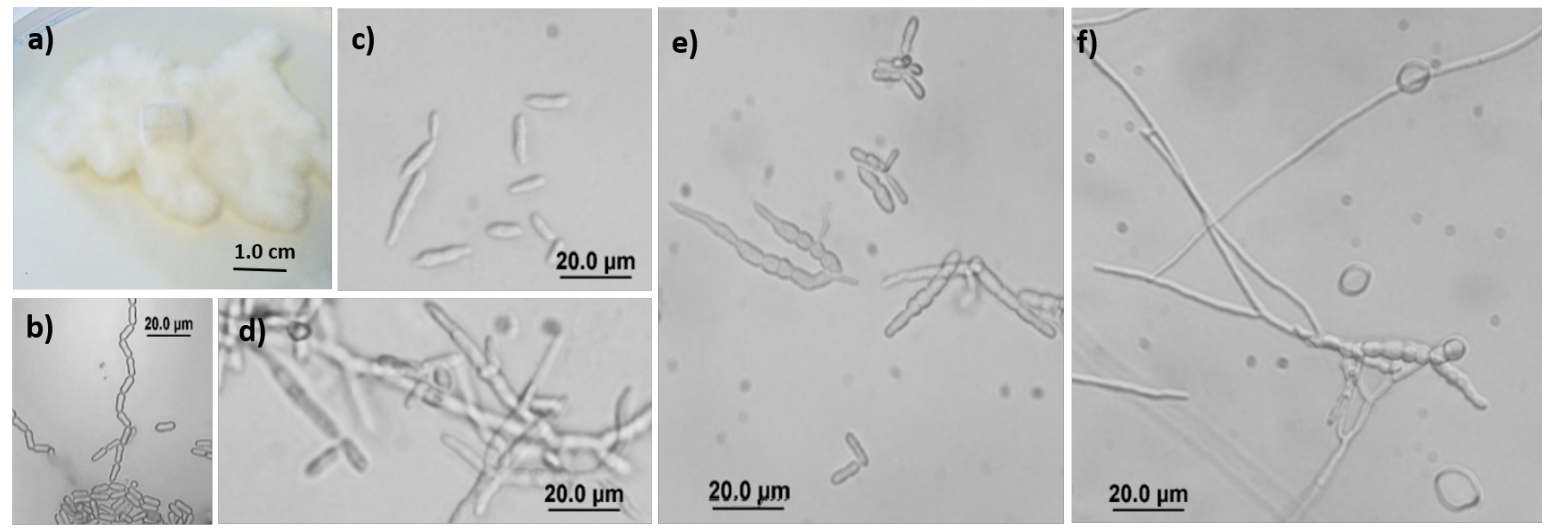

Fig. 3 Characteristics and morphological development patterns of blastospores of Metarhizium sp. 02. The liquid culture was grown in GICM supplemented with $10 \% \mathrm{FBS}$ and incubated at $28^{\circ} \mathrm{C}$ under static condition. (a) Colony of Metarhizium sp. 02 on PDA incubated at $28^{\circ} \mathrm{C}$ for 7 days; (b) conidia; (c,d) blastospore formation after 2 days of incubation; (e,f) germination and elongation of blastospores after 3 days of incubation.
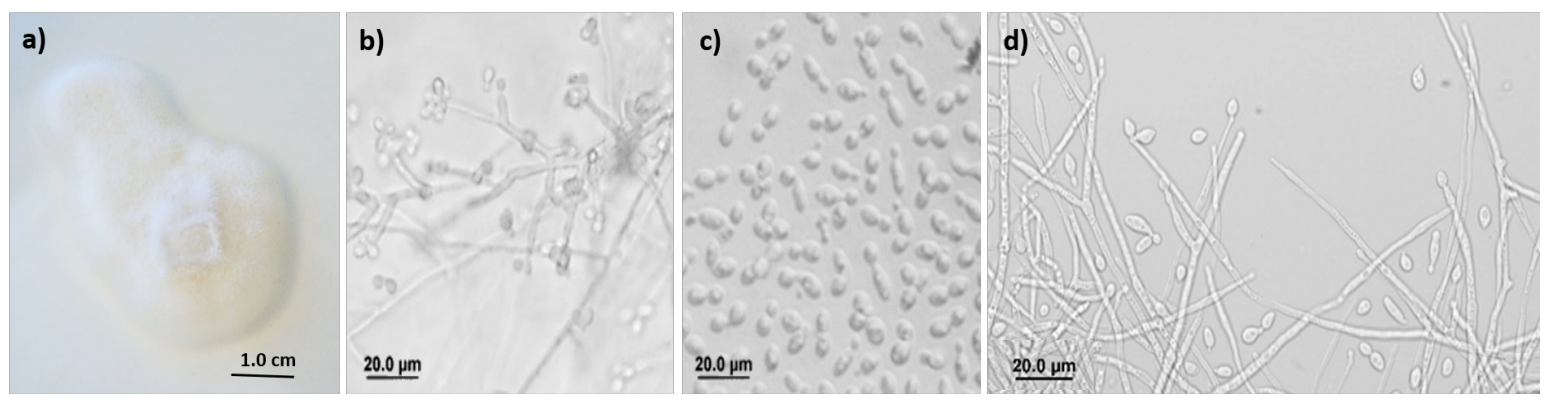

Fig. 4 Characteristics and morphological development patterns of blastospores of $A$. pistillariiformis. The liquid culture was grown in GICM supplemented with $10 \%$ FBS and incubated at $28^{\circ} \mathrm{C}$ under static condition. (a) Colony of A. pistillariiformis on PDA incubated at $28^{\circ} \mathrm{C}$ for 7 days; (b) blastospore formation after 2 days of incubation; (c,d) released blastospores.

curred, followed by a production of germ tubes after 3 days of incubation. A cross wall was subsequently made to separate part of the stretched blastospores. During this stage, shape of blastospores were transformed to tamarind-like shapes and size was increased simultaneously (Fig. 3e). After 5 days of cultivation, germinated blastospores became short mycelia and continued to grow slowly in the GICM. However, formation of new blastospores was not detected in this study (Fig. 3f).

Members in the genus Akanthomyces can produce valuable toxins and metabolites. Colony appearance of $A$. pistillariiformis grown on PDA at $28^{\circ} \mathrm{C}$ for one week was flat and wet on the surface with approximately $12.78 \pm 2.05 \mathrm{~mm}$ in diameter (Fig. 4a). Colony color was cream to white and yellow on the reverse side. Mycelia grew very slowly in the liquid medium. Blastospores were observed after 2 days of cultivation, which appeared laterally or sometimes terminally from mycelia (Fig. 4b). They were hyaline, single-celled with smooth-wall with varying shapes from round to oval (Fig. 4c). The average size of blastospores was 4.3-8.1 $\mu \mathrm{m} \times 3.9-$ $4.3 \mu \mathrm{m}$ and usually produced from hyphal tips and formed clusters. After released into the medium, blastospores began to bud immediately (Fig. 4c). However, new budding cells did not detach from the mother cells which were not the case in $C$. brongniartii and Hirsutella sp. 02 . No germination of blastospores was observed within 14-day period of observation (Fig. 4d).

According to the observation of developmental process of blastospores, common characteristics of blastospores among the isolates can be summarized. Generally, blastospores are single cells, hyaline, and non-motile with smooth thin-walled resembling protoplast-like cells. They propagated by bursting through a pore on a hyphal fragment and connected 
to the mycelium through a short neck. Once ramification of mycelium has taken place under suitable environmental conditions, formation of blastospore was induced. After disconnected, blastospores enlarged in size and shape, followed by initiation of germination to form new mycelia. However, blastospores of some genera underwent through a process of budding similar to that of yeasts.

\section{Effects of media, types of inoculum and cultivating conditions on the production of blastospores}

Optimal condition for the production of blastospores of C. brongniartii, Hirsutella sp. 02, Metarhizium sp. 02, and A. pistillariiformis were determined. Effects of media, types of inoculum and cultivating conditions were examined for the maximum production of blastospores. Two different media (GICM and YPG) were tested. The results showed that blastospore production of $C$. brongniartii was induced by cultivation in both GICM and YPG liquid media (Fig. 5a). The GICM gave a higher yield of blastospores compared to that of YPG regardless of types of inoculum and cultivating conditions. Meanwhile blastospore production of Hirsutella sp. 02, Metarhizium sp. 02 and A. pistillariiformis could only be induced by cultivation in GICM. Therefore, the GICM was used as culture medium in all subsequent experiments in this study.

Two different types of inoculum (mycelial plug and conidia suspension) were also tested. The results showed that conidia suspension gave a slightly higher yield of blastospores than that of mycelial plug when cultivated in the GICM for all tested fungal isolates (Fig. $5 \mathrm{a}-\mathrm{d}$ ). Numbers of blastospores produced from both types of inoculum were not significantly different according to Duncan's multiple range test $(p<0.05)$. Therefore, mycelial plugs were used as a starting inoculum for the production of blastospores due to cost and ease of preparation. Different cultivating conditions, shaking and static were subsequently evaluated. Regardless of inoculum types and culture media, production rate of C. brongniartii blastospores under static condition increased slowly; and the numbers of blastospores obtained were lower when compared to those under shaking condition. However, cultivating conditions were species-specific as no blastospore was observed under shaking condition from Hirsutella sp. 02, Metarhizium sp. 02 and A. pistillariiformis cultures.

Time course for the production of blastospores was observed in all entomopathogenic fungal isolates. In the $C$. brongniartii, blastospore formation initiated at 12-18 hours post inoculation (hpi) and yield of blastospores reached the maximum level at 36-72 hpi. After 78 hpi of cultivation, most of blastospores started to germinate. Regarding cultivating conditions, the highest yield of blastospores was $2.43 \times 10^{6}$ blastospores/ $\mathrm{ml}$ under static condition when either conidia or mycelial plugs were used as inoculum in GICM. On the other hand, the highest yield of blastospores under shaking condition was $3.28-4.35 \times 10^{8}$ blastospores $/ \mathrm{ml}$ when either conidia or mycelial plugs were used as inoculum in GICM. It can be thus concluded that the best conditions for the production of blastospore of C. brongniartii was using GICM as the induction medium under shaking condition. The suitable time course for harvesting fresh blastospores, to be used as specimens for cryopreservation, was approximately at 42-72 hpi after incubation.

In Hirsutella sp. 02, blastospore formation was initiated at $36 \mathrm{hpi}$ and numbers of blastospores were exponentially increased within a day. A sudden decrease in spore production rate was subsequently detected, followed by second period of exponential increase in numbers of blastospores. This interesting observation indicated a unique pattern of blastospore development of Hirsutella sp. 02 in the GICM. The sudden change in the numbers of blastospores at $84 \mathrm{hpi}$ could arise from the fact that majority of budding blastospores were detached from one another, resulting in an increased numbers of blastospores. Blastospore production reached the maximum level at 96-132 hpi, yielding $4.8 \times 10^{6}$ blastospores $/ \mathrm{ml}$. A decline in blastospore numbers occurred immediately due to rapid germination of most blastospores. According to the results, the best conditions for the production of blastospore of Hirsutella sp. 02 were cultivating in GICM under static condition. The suitable time course for harvesting fresh blastospores was approximately at 84-144 hpi.

In Metarhizium sp. 02, blastospore formation was initiated at $48 \mathrm{hpi}$. The exponential increase in blastospore numbers was observed within $72 \mathrm{hpi}$ as budding blastospores were detached from one another. The highest numbers of blastospores was observed at $108 \mathrm{hpi}$, yielding $4.4 \times 10^{6}$ blastospores $/ \mathrm{ml}$. A sharp decline in blastospore numbers was observed after 4 days of incubation resulting from rapid formation of emerging young mycelia. It can be thus concluded that the best conditions for the production of blastospores of Metarhizium sp. 02 were using GICM as the induction medium under static condition. The suitable time course for harvesting fresh blastospores 

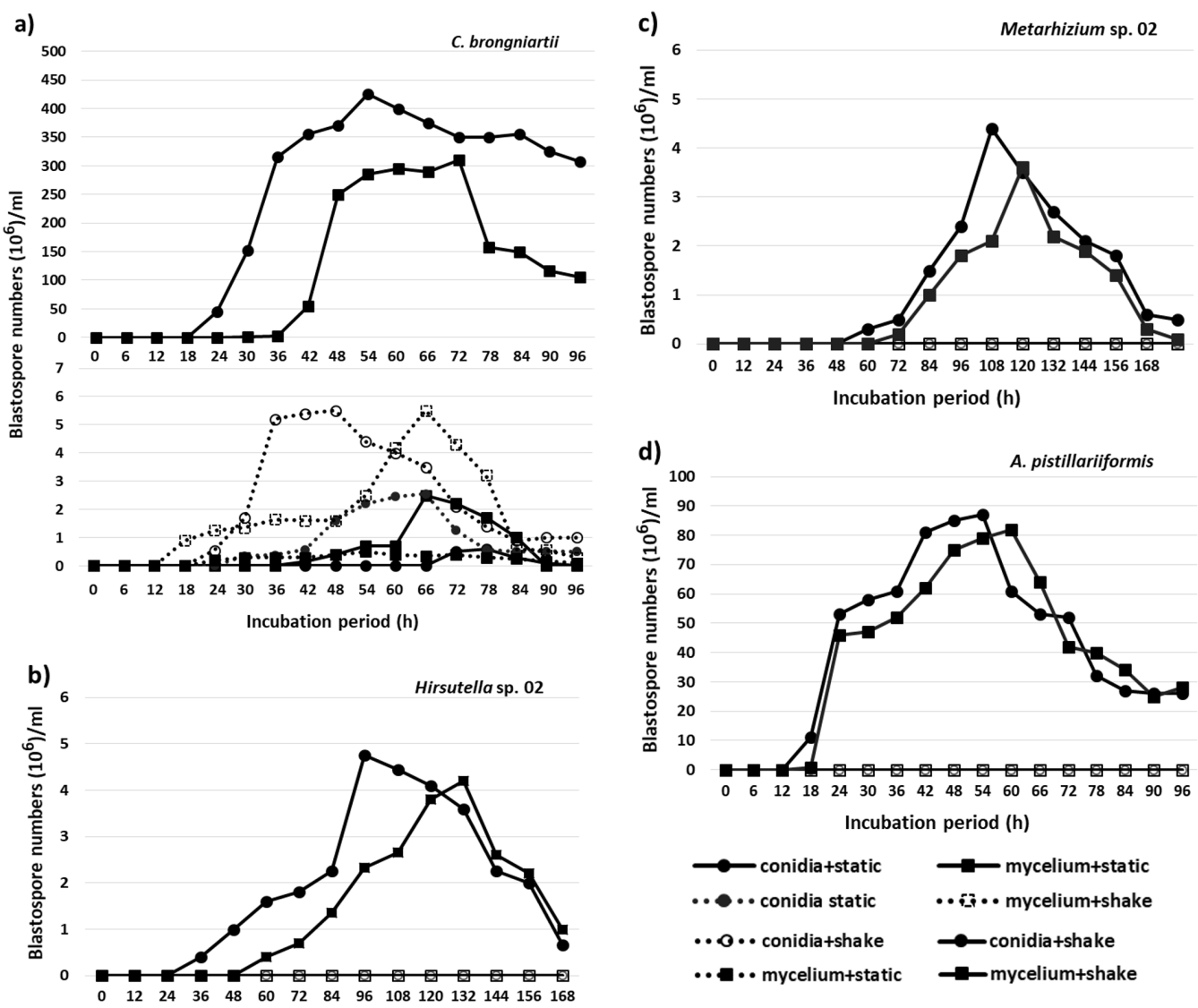

Fig. 5 Production of blastospores of selected entomopathogenic fungi incubated at $28^{\circ} \mathrm{C}$ under shaking and static conditions. Mycelial plugs and conidia were used as stating inocula. (a) Production of blastospores of $C$. brongniartii in YPG (dotted line) and GICM (solid line); (b) production of blastospores of Hirsutella sp. 02 in GICM; (c) production of blastospores of Metarhizium sp. 02 in GICM; (d) production of blastospores of A. pistillariiformis in GICM. The results are presented as the mean \pm SD of three independent experiments.

was approximately at 84-144 hpi. In A. pistillariiformis, blastospore formation was initiated at $12 \mathrm{hpi}$ and numbers of blastospores were exponentially increased up to $48 \mathrm{hpi}$, yielding $8.8 \times 10^{7}$ blastospores $/ \mathrm{ml}$. Therefore, the best conditions for blastospore production of $A$. pistillariiformis were cultivating in GICM under static condition. The suitable time course for harvesting fresh blastospores was approximately at 24-72 hpi after incubation.

\section{Cryopreservation of blastospores of entomopathogenic fungi}

Long-term preservation of blastospores of the entomopathogenic fungi was studied. Freeze-drying was used as a preservation method and $5 \%(\mathrm{w} / \mathrm{v})$ trehalose was used as a protective agent. Viability
Table 3 Viability of four entomopathogenic fungi using freeze-drying method.

\begin{tabular}{lcc}
\hline \multirow{2}{*}{ Storage } & \multicolumn{2}{c}{ Viability (\%) } \\
\cline { 2 - 3 } & Preservation & One-year storage \\
\hline C. brongniartii & $31.8 \pm 3.4$ & $21.8 \pm 1.8^{\mathrm{a}}$ \\
Hirsutella sp. 02 & $41.8 \pm 2.2$ & $35.6 \pm 0.9^{\mathrm{b}}$ \\
Metarhizium sp. 02 & $31.8 \pm 2.2$ & $22.0 \pm 1.7^{\mathrm{c}}$ \\
A. pistillariiformis & $0.00 \pm 0.0$ & $0.00 \pm 0.0$ \\
\hline
\end{tabular}

The results are presented as the mean value $\pm S D$ of three independent experiments. Different letters indicate significant differences according to Duncan's multiple range test $(p<0.05)$.

of blastospores was assessed by determination of germ tube formation assay immediately after preser- 
vation and after one-year storage at $5{ }^{\circ} \mathrm{C}$ in the dark. The preservation results revealed that freezedrying method could be used to preserve fungal blastospores of C. brongniartii, Hisutella sp. 02 and Metarhizium sp. 02 because satisfactory percentage of viability was obtained after one-year storage, as shown in Table 3. However, the method was not suitable for preserving blastospores of $A$. pistillariiformis since no viability was observed. Observation of revived cells in terms of morphological and genetic stability demonstrated no changes in pigmentation, colony morphology including ability to produce blastospores and reproductive structures.

\section{DISCUSSION}

Entomopathogenic fungi in the genera Akanthomyces, Cordyceps, Hirsutella, Metarhizium, and Torrubiella have been recognized as potential candidates for the production of microbial insecticides to control several agricultural pests and have been identified as valuable sources of bioactive metabolite production. They are considered agriculturally and medically important. Therefore, understanding the morphological development of entomopathogenic fungi is one of the key parameters prior to using blastospores in industrial production. In addition, preservation methods which maintain genetic and physical stabilities of entomopathogenic fungi, were required to avoid alterations during storage.

In the present study, production of entomopathogenic fungal blastospores could be induced by using GICM as a culture medium supplemented with FBS. However, induction of blastospores in vitro depends on fungal genera since the findings revealed that none of the isolates in the genus Torrubiella could produce blastospores. Similarly, production of blastospores also depends on fungal isolates since blastospore formation could be detected in some isolates of the genera Akanthomyces, Cordyceps, Hirsutella and Metarhizium with different efficiency. As blastospore formation has been reported as an indispensable phase in the fungal reproducing cycle [17], failure in the production of blastospores in some fungal isolates could be attributed to inappropriate conditions of cultivation used.

Out of 39 entomopathogenic fungal isolates, 18 isolates were able to produce blastospores. Most of them belonged to the genera Cordyceps and Hirsutella. Most isolates of Cordyceps spp. were capable to produce blastospores (63\%) which suggested that Cordyceps spp. is non-fastidious and does not require complex nutrients for growth and development. Common characteristics of blastospores among those four genera were single-cell, hyaline, non-motile with smooth thin-walled, and varied in size and shape. Development patterns of blastospore formation also depends on fungal genera in term of day of spore initiation, spore emerging position, spore budding, and spore germination. It can be noted that blastospore morphology and development of Akanthomyces sp. is unique among other genera. This characteristic could be used to distinguish Akanthomyces sp. from other fungal genera. This corresponds to a statement from Samson et al [9] who stated that blastospores may be used to classify a genus of entomopathogenic fungi in the same manner as other vegetative propagules or other structures.

Culture media, types of inoculum and cultivating conditions exhibited different impacts on the production of blastospores. The GICM supplemented with FBS could be used to induce the production of blastospores in all fungal genera tested, however, YPG medium could only be used to induce the production of blastospores in the genus Cordyceps (C. brongniartii). FBS acts to mimic natural condition of haemolymph and contains essential components, such as hormones, vitamins, minerals, trace elements, and growth factors [18], which contributed to the induction of blastospore formation of entomopathogenic fungi in vitro. An ability of $C$. brongniartii for production blastospores in YPG medium might be attributed from nonfastidious character of the fungus including strong virulence resembling to its anamorph, $B$. brongniartii. This ability could thus be one of the factors contributing to adaptation ability to diverse environmental conditions. YPG medium was also used to promote the production of blastospores in $B$. bassiana and C. brongniartii under shaking condition, yielding $8.5 \times 10^{7}$ blastospores $/ \mathrm{ml}$ and $5.7 \times 10^{6}$ blastospores $/ \mathrm{ml}$, respectively, after 2 days of incubation [19].

In addition to culture media, either mycelial plugs or conidia suspensions could be used as starting inocula since the numbers of blastospores produced from both were not significantly different. Cultivating conditions also showed different impacts on blastospore production because cultivation under static condition was only suitable for Hirsutella sp. 02, Metarhizium sp. 02 and A. pistillariiformis, while $C$. brongniartii produced blastospores in both static and shaking conditions. Production of blastospores under shaking condition has been 
described as a suitable condition for the production of blastospores in several entomopathogenic fungi, for example M. flavoviride, B. bassiana, P. fumosoroseus, and C. unilateralis) [20-23]. However, in the present study, production of blastospores of Hirsutella sp. 02, Metarhizium sp. 02 and A. pistillariiformis was only successful under static condition. Furthermore, those parameters tested also had significant impacts on time course of blastospore formation and determination of cultivation period that generated the highest numbers of blastospores. The observation revealed that initiation of blastospore formation of $C$. brongniartii occurred faster than other isolates. This might be a result from the fact that this fungus is fast-growing when grown on PDA and in GICM while the others are slowgrowing.

The findings provided not only characteristics and development patterns of blastospores of entomopathogenic fungi, but also demonstrated the requirements for specific nutrients and conditions for growth and development of individual isolate. Some fungal species might need different types of sugars or nitrogen sources in medium composition, which could affect the numbers of blastospore production, growth and morphology. Formation of blastospores might be affected by age of the culture and loss of virulence due to routine subculturing. As blastospores serve as a virulent determinant in entomopathogenic fungi, loss of virulence due to routine subculturing may result in loss of ability to produce blastospores accordingly.

Long-term preservation of blastospores using freeze-drying method was successful in C. brongniartii, Hisutella sp. 02 and Metarhizium sp. 02. However, the method was not suitable to preserve blastospores of $A$. pistillariiformis because no viability was observed. This finding might suggest different compositions and properties of blastospores of Akanthomyces spp., which make the fungus more sensitive to freeze-drying. This, consequently, leads to a problem in preservation of this fungus. Therefore, further investigation to identify an appropriate technique, conditions and protective agents must be conducted for this genus. In addition, several reports described that selection of optimal growth phase for cryopreservation is also essential for survival of dried spores. For example, the stationary phase cells of Lactobacillus rhamnosus gave the highest recovery, after drying, of $31-50 \%$, whereas early log phase cells exhibited 14\% survival and lag phase cells showed the highest susceptibility, with only $2 \%$ cell survival [24]. Similar results were reported by Mary et al [25], where significantly higher cell viability was achieved from stationary phase Rhizobia cells compared to exponential phase cells. In contrast, higher survival rates of Sinorhizobium and Bradyrhizobium were produced when sampled in the lag phase of growth [26]. According to the finding from Cliquet and Jackson [20], a defined medium containing basal salts, glutamate, glucose, and zinc can be used to induce optimal concentrations of desiccation-tolerant blastospores of $P$. fumosoroseus. However, in this study the growth phase of entomopathogenic fungi was not determined but only the time course for maximum production of blastospores.

\section{Appendix A. Supplementary data}

Supplementary data associated with this article can be found at http://dx.doi.org/10.2306/ scienceasia1513-1874.2020.078.

Acknowledgements: This work was supported by the National Science and Technology Development Agency (NSTDA), Thailand and grants from Prince of Songkla University Research and Development Office for Lakkhana Kanhayuwa Wingfield.

\section{REFERENCES}

1. Thomas KC, Khachatourians GG, Ingledew WM (1987) Production and properties of Beauveria bassiana conidia cultivated in submerged culture. Can J Microbiol 33, 12-20.

2. Peng G, Wang Z, Yin Y, Zeng D, Xia Y (2008) Field trials of Metarhizium anisopliae var. acridum (Ascomycota: Hypocreales) against oriental migratory locusts, Locusta migratoria manilensis (Meyen) in Northern China. Crop Prot 27, 1244-1250.

3. Mascarin GM, Lopes RB, Delalibera I, Fernandes EKK, Luz C, Faria M (2019) Current status and perspectives of fungal entomopathogens used for microbial control of arthropod pests in Brazil. J Invertebr Pathol 165, 46-53.

4. Jiang W, Peng Y, Ye J, Wen Y, Liu G, Xie J (2020) Effects of the entomopathogenic fungus Metarhizium anisopliae on the mortality and immune response of Locusta migratoria. Insects 11, ID 36.

5. Petlamul W, Boukaew S, Hauxwell C, Prasertsan P (2019) Effects on detoxification enzymes of Helicoverpa armigera (Lepidoptera: Noctuidae) infected by Beauveria bassiana spores and detection of its infection by PCR. ScienceAsia 45, 581-588.

6. Isaka $M$, Tanticharoen $M$, Thebtaranonth $Y$ (2000) Cordyanhydrides $\mathrm{A}$ and $\mathrm{B}$, two unique anhydrides from the insect pathogenic fungus Cordyceps pseudomilitaris BCC 1620. Tetrahedron Lett 41, 1657-1660. 
7. Isaka M, Tanticharoen M (2001) Structures of cordypyridones A-D, antimalarial n-hydroxy- and nmethoxy-2-pyridones from the insect pathogenic fungus Cordyceps nipponica. J Org Chem 66, 4803-4808.

8. Kittakoop P, Punya J, Kongsaeree P, Lertwerawat Y, Jintasirikul A, Tanticharoen M, Thebtaranonth Y (1999) Bioactive naphthoquinones from Cordyceps unilateralis. Phytochem 52, 453-457.

9. Rachmawati R, Kinoshita H, Nihira T (2018) Production of insect toxin beauvericin from entomopathogenic fungi Cordyceps militaris by heterologous expression of global regulator. J Agric Sci 40, 177-184.

10. Samson RA, Evans HC, Patgé JP (1988) Atlas of Entomopathogenic Fungi, Springer, Berlin.

11. Pendland JC, Hung SY, Boucias DG (1993) Evasion of host defense by in vivo-produced protoplast-like cells of the insect mycopathogen Beauveria bassiana. $J$ Bacteriol 18, 5962-5969.

12. Inglis DG, Goettel MS, Butt TM, Strasser H (2001) Use of Hyphomycetous fungi for managing insect pests. In: Butt TM, Jackson C, Magan N (Eds) Fungi as Biocontrol Agents: Progress, Problems and Potential, CABI Publishing, pp 23-51.

13. Jackson MA, McGuire MR, Laccy LA, Wraight SP (1997) Liquid culture production of desiccation tolerant blastospores of the bioinsecticidal fungus Paecilomyces fumosoroseus. Mycol Res 101, 35-41.

14. Vega FE, Jackson MA, McGuire MR (1999) Germination of conidia and blastospores of Paecilomyces fumosoroseus on the cuticle of the silverleaf whitefly, Bemisia argentifolii. Mycopathologia 147, 33-35.

15. Corrêa B, da Silveira Duarte V, Silva DM, Mascarin GM, Delalibera Júnior I (2020) Comparative analysis of blastospore production and virulence of Beauveria bassiana and Cordyceps fumosorosea against soybean pests. BioControl 65, 323-337.

16. Deshpande MV (1999) Mycopesticide production by fermentation: potential and challenges. Crit Rev Microbiol 25, 229-243.
17. Beauvais A, Latgé JP (1989) Chitin and $\beta(1-3)$ glucan synthases in the protoplastic entomophthorales. Arch Microbiol 152, 229-236.

18. Van Der Valk J, Mellor D, Brands R, Fischer R, Gruber F, Gstraunthaler G et al (2004) The humane collection of fetal bovine serum and possibilities for serumfree cell and tissue culture. Toxicol In Vitro 18, 1-12.

19. Bidochka MJ, Pfeifer TA, Khachatourians GG (1987) Development of entomopathogenic fungus Beauveria bassiana in liquid culture. Mycopathologia 99, 77-83.

20. Cliquet S, Jackson MA (1999) Influence of culture conditions on production and freeze-drying tolerance of Paecilomyces fumosoroseus blastospore. $J$ Ind Microbiol Biotechnol 23, 97-102.

21. Wongsa P, Tasanatai S, Watts P, Hywel-Jones N (2005) Isolation and in vitro cultivation of the insect pathogenic fungus Cordyceps unilateralis. Mycol Res 109, 936-940.

22. Kocharin K, Wongsa P (2006) Semi-defined medium for in vitro cultivation of the fastidious insect pathogenic fungus Cordyceps unilateralis. Mycopathologia 161, 255-260.

23. Ying SH, Feng MG (2006) Novel blastosporebased transformation system for integration of phosphinothricin resistance and green fluorescence protein genes into Beauveria bassiana. Appl Microbiol Biotechnol 72, 206-210.

24. Corcoran BM, Ross RP, Fitzgerald GF, Stanton C (2004) Comparative survival of probiotic lactobacilii spray-dried in the presence of prebiotic substances. $J$ Appl Microbiol 96, 1024-1039.

25. Mary P, Ochin D, Tailliez R (1986) Growth status of rhizobia in relation to their tolerance to low water activities and desiccation stresses. Soil Biol Biochem 18, 179-184.

26. Boumahdi M, Mary P, Hornez JP (1999) Influence of growth phases and desiccation on the degrees of unsaturation of fatty acids and the survival rates of Rhizobia. J Appl Microbiol 87, 611-619. 


\section{Appendix A. Supplementary data}

Table S1 General characteristics and blastospore formation patterns of the entomopathogenic fungi.

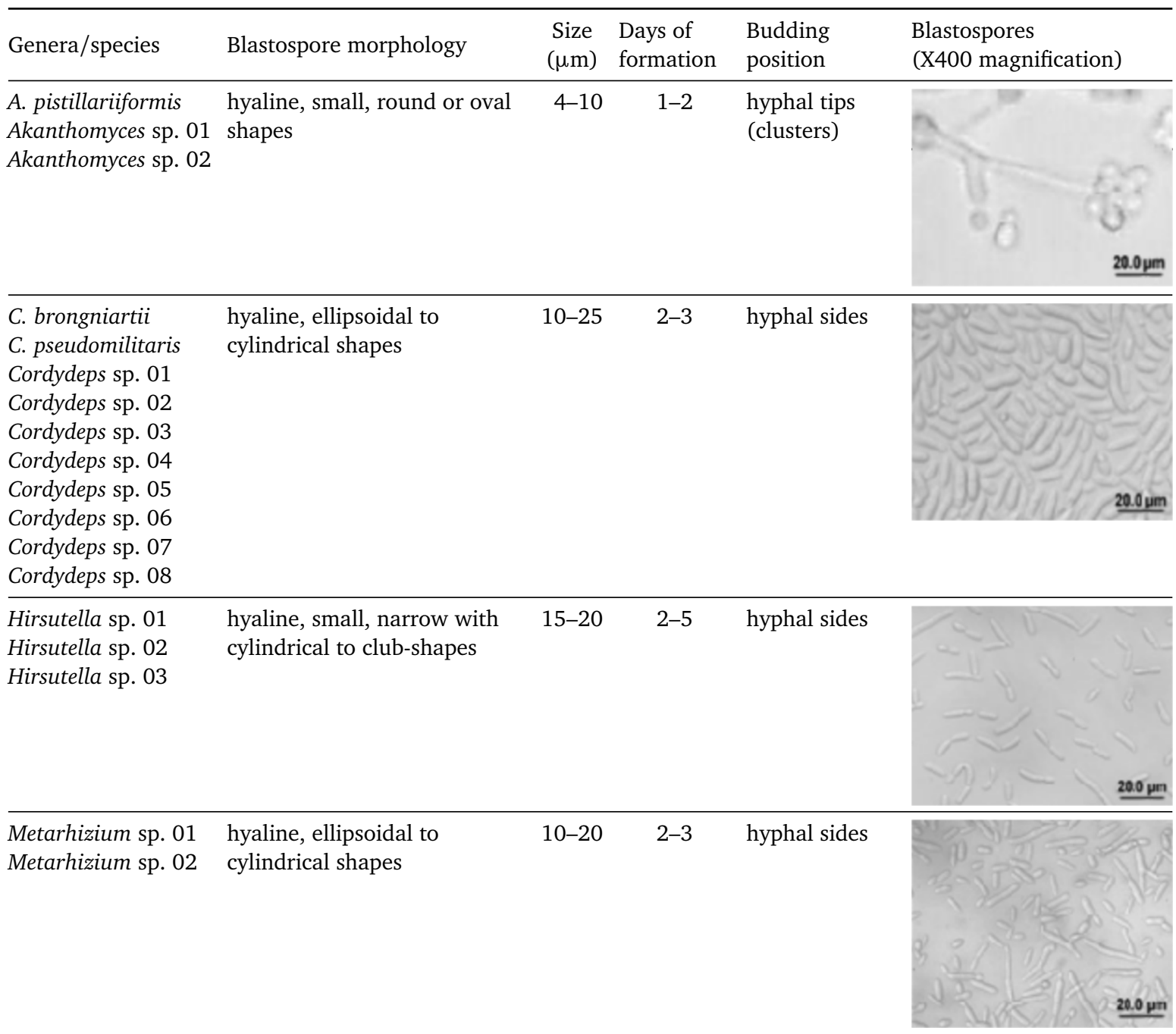

